\title{
How Straight Do Axons Grow? ${ }^{1}$
}

\author{
MICHAEL J. KATZ \\ Neurobiology Center and Department of Biometry, Case Western Reserve University, Cleveland, Ohio 44106
}

\begin{abstract}
Detailed growth paths of embryonic frog and chick axons were measured as the axons elongated in dispersed cultures on acid-rinsed glass surfaces. Mathematical analyses demonstrate that, under these conditions, axons do not grow randomly but tend to grow straight. Growth cones appear to actively alternate sides - right and left from the straight line of growth - and the growth cone neck exhibits all possible angles, but the axon itself maintains a fairly constant orientation. It appears that an axonal resistance to bending may be the cause of the intrinsic tendency for relatively straight axonal growth. The natural straightness of axonal growth may be an important developmental determinant of certain in vivo axon patterns.
\end{abstract}

Within an organism, axons are found in a great many different configurations, spanning a range from straight lines to hairpin loops (Ramón y Cajal, 1911). In vitro studies have demonstrated that the configuration assumed by an axon can be completely determined by the pattern of local extrinsic features in the substrate through which the axon grows. For example, axons will follow the contours of the most adhesive substrate, even when those contours have acute bends (Weiss, 1945; Letourneau, 1975a; Collins and Garrett, 1980). Likewise, in vivo studies are also consistent with the idea that the configuration of an axon within an organism can be almost entirely determined by extrinsic factors, especially the contours of its local environment (e.g., Ramón y Cajal, 1911; Speidel, 1933; Rakic, 1971; Katz and Lasek, 1979; Silver and Sidman, 1980; Katz, 1984).

In contrast, a number of in vitro and in vivo studies have suggested that, in some situations, factors intrinsic to the neuron may mold the configuration of its axon (Bray, 1973; Strassman et al., 1973; Heacock and Agranoff, 1977; Lasek et al., 1983; Katz, 1984; Katz and Lasek, 1985a, b). If a neuron could be grown in an ideal homogeneous environment-an environment with no extrinsic guiding contours-then its axon should assume a configuration that was largely determined by intrinsic factors. Tissue culture systems can provide relatively homogeneous environments, and by growing axons in dispersed culture on homogeneous surfaces, one may be able to reveal the intrinsic determinants of axon configurations.

From such tissue culture studies, Bray (1979) and others have

Received April 23, 1984; Revised August 27, 1984;

Accepted August 31, 1984

'I am indebted to W. F. Eddy for many constructive conversations about the problems of analyzing the straightness of growth of axons, to T. Hoshiko for reminding me about fractals, to R. J. Lasek for first raising the question of how straight axons actually grow, and to L. J. Gilbert and L. F. Watson for excellent technical assistance. This work was supported by grants from the National Institutes of Health and the Whitehall Foundation and by a Sloan Foundation Research Fellowship. shown that an important intrinsic factor determining the final axon configuration is a straightening tendency. Due to internal tensions, axons tend to form the shortest straight lines between attachment points. For this reason, established axon cultures are usually composed of fairly straight segments of axon. Beyond this elastic straightening, however, it is possible that axons actually grow in relatively straight paths (Harrison, 1910; Bray, 1973, Trinkaus, 1984). The present study follows axons as they are actively elongating and attempts to rigorously document the extent to which axons have an inherent tendency to grow straight.

\section{Materials and Methods}

\section{Cultures}

Frog neurons. Xenopus embryos were obtained from matings of adult frogs; matings were induced by injection of human chorionic gonadotropin (Sigma Chemical Co.). Twenty-four- to 48-hr-old amphibian primary tissue cultures were grown by disaggregating neural tube cells of tailbud stage Xenopus embryos and plating these cells on acid-rinsed glass coverslips set in the bottoms of 35-mm sterile Falcon Petri dishes (Spitzer and Lamborghini, 1976). (Polyornithine-coated coverslips were made according to the method of Letourneau (1975a) (poly-L-ornithine, ICN Nutritional Biochemicals).) Stage 28-30 (Nieuwkoop and Faber, 1967) Xenopus embryos were removed from their vitelline membranes and washed through four changes of sterile Steinberg solution, pH 7.5 (Hamburger, 1960). Next, the ectoderms were peeled off and the brain primordia were dissected free and washed in fresh Steinberg solution. Brain primordia were then disaggregated by soaking them for 10 $\mathrm{min}$ in a calcium- and magnesium-free medium $(59 \mathrm{~mm} \mathrm{NaCl}, 0.7 \mathrm{~mm} \mathrm{KCl}$, $0.4 \mathrm{mM}$ EDTA, pH 7.5). Cell aggregates were broken apart by pipetting briefly at 5-min intervals with a fire-polished Pasteur pipette. Cell suspensions were then plated in fresh Steinberg solution supplemented with $2 \%$ fetal bovine serum, 5 to $10 \mu \mathrm{M}$ nerve growth factor (NGF), and antibiotics (50 units/ml of penicillin, $0.05 \mathrm{mg} / \mathrm{ml}$ of streptomycin; Grand Island Biological Co.).

Approximately 1 to 1.5 brain primordia were plated per $35-\mathrm{mm}$ culture dish and grown at $21^{\circ} \mathrm{C}$. Under these conditions, 24-hr cultures averaged 150-170 differentiated cells/22 $\times 22 \mathrm{~mm}$ coverslip, and approximately onefourth of these cells were neurons.

Chick neurons. Twenty-four- to 48-hr-old avian primary tissue cultures were grown from dorsal root ganglion cells of 7- to 12-day chick embryos (Shaw and Bray, 1977). Cells were isolated by treating ganglia with trypsin $(2.5 \mathrm{mg} / \mathrm{ml})$ and collagenase $(0.5 \mathrm{mg} / \mathrm{ml})$ at $37^{\circ} \mathrm{C}$ for $25 \mathrm{~min}$ in calcium- and magnesium-free Hanks' solution and then disaggregating the cells by passing them through a small-bore pipette. Cultures of isolated cells were enriched for neurons by differential adhesion. First, the cells were grown for 30 to 40 min in Leibovitz's L-15 medium supplemented with $0.6 \%$ glucose and antibiotics. Next, the medium was replaced with complete culture medium, and the dish was agitated on a vortexer to free the loosely adherent neurons. The resultant cell suspension was plated on acid-washed glass coverslips that had been sealed over a $1.5-\mathrm{cm}$ hole drilled in the bottom of a $35-\mathrm{mm}$ culture dish. Finally, fresh culture medium was added. The complete culture medium consisted of Leibovitz's L-15 medium (Grand Island Biological Co.) with $10 \%$ fetal bovine serum, $0.6 \%$ glucose, $0.3 \%$ methylcellulose, 5 to 10 $\mu \mathrm{M} \mathrm{NGF}, 100$ units $/ \mathrm{ml}$ of penicillin, $100 \mu \mathrm{g} / \mathrm{ml}$ of streptomycin, and $0.3 \mu \mathrm{g} /$ $\mathrm{ml}$ of arabinosylcytosine added. 


\section{Data collection}

Criteria for choosing axons. Axons selected for study were only from clearly healthy cultures and were in areas of minimal debris. The axons were all at least $100 \mu \mathrm{m}$ long and were separated from other cells and cell processes by at least $100 \mu \mathrm{m}$. Data were analyzed only for axons that grew without major retractions and without contacting other cells.

Definition of standard axonal sites. At the end of each time period, coordinates were measured at five sites along each axon (Figs. 1 and 2). The growth cone site was taken to be the center of the palm-like growth cone. The neck site was taken to be the constriction or the point of sharp angle at the proximal end of the growth cone. (A clear neck point could only

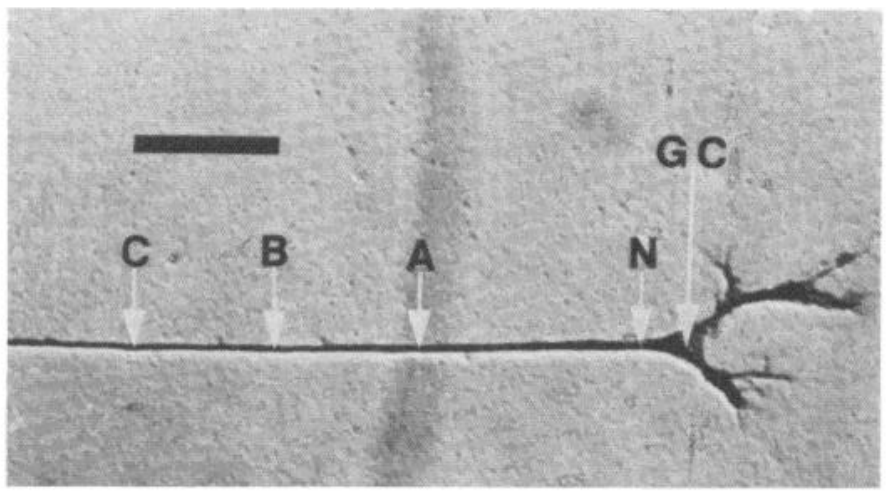

Figure 1. Micrograph of fixed chick dorsal root ganglion axon in culture, indicating sites of measurement $(C, B, A$, and $N)$ cf. Figure 2. Bar $=10 \mu \mathrm{m}$. Bodian stain with intensifier (see Katz and Watson, 1984).

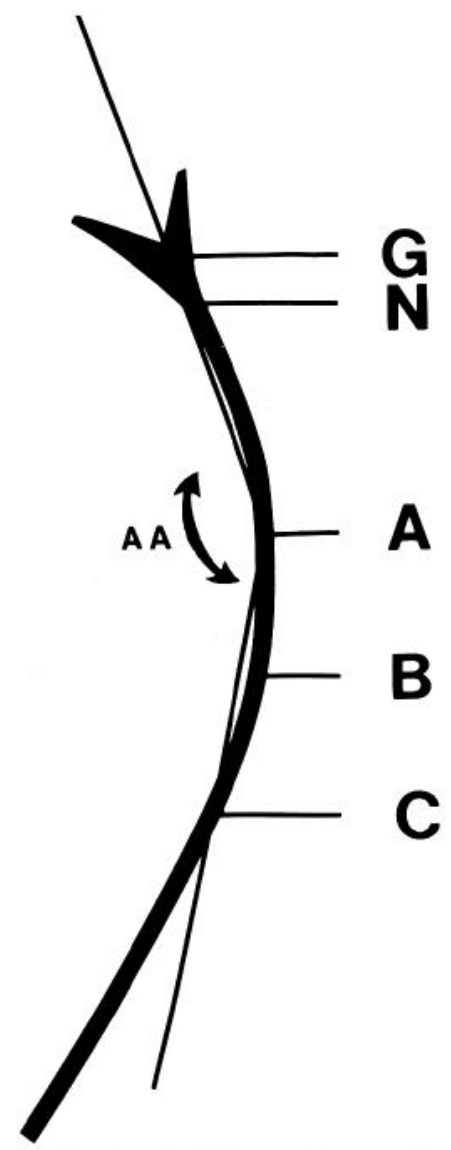

Figure 2. Drawing of the terminal $50 \mu \mathrm{m}$ of an axon indicating five standard sites $(G, N, A, B$, and $C)$ of measurement during quantification of axonal growth. $A A$, angle of bend of the terminal $40 \mu \mathrm{m}$ of axon. See the text for details. be identified about half of the time.) Three additional sites were located more proximally along the axon, using the growth cone site as the initial point of reference: point $\mathrm{A}$ was taken to be $20 \mu \mathrm{m}$ along the axon proximal to the growth cone, point $\mathrm{B}$ was taken to be $30 \mu \mathrm{m}$ along the axon proximal to the growth cone, and point $\mathrm{C}$ was taken to be $40 \mu \mathrm{m}$ along the axon proximal to the growth cone.

Measurement procedures. Axonal growth was monitored at $\times 400$ magnification on an Olympus IMT inverted phase microscope to which was attached an RCA TC2000 Newvicon video camera equipped with an Olympus FK $3.3 \times$ projection eyepiece. Time lapse video records were made on Sony BR video cassettes ( $3 / 4$-inch tape) using an NEC video recorder (VC9507 ) running at $1 / 64$ th real time. Real time was indicated with a VICOM V240 date/time generator. The locations of the standard axonal sites were measured on a transparent grid that overlay the video screen (Panasonic WV5300 ). The standard units of measurement were marked on a flexible plastic template which was placed on the screen to accurately measure distances along the curved axons. The screen had a diagonal length of approximately $21 \mathrm{~cm}$ and the grid was divided into 5 squares $/ \mathrm{cm}$; in our system this resulted in a scale of $2.56 \mu \mathrm{m} / \mathrm{square}$ for the final video image. Data were collected by stopping (freezing) the tape at an exact time point and then measuring the simultaneous locations of the five standard axonal sites. Figure 3 presents reconstructions of the growth cone movements of four axons recorded in this manner.

Precision of measurements. A static image (a calibrated stage micrometer) was recorded for $2 \mathrm{hr}$, rotated $90^{\circ}$, and then recorded for an additional $2 \mathrm{hr}$. The image did not drift, and distances remained undistorted by the rotation. Locations could be read from the screen to an accuracy of $1 / 3$ grid square $(\sim 1 \mu \mathrm{m})$. However, by repeating readings for 80 separate time points on data tapes of growing axons, we found our overall average error to be closer to $2 \mu \mathrm{m}$.

\section{Computations}

Straightness of growth. As a standardized measure of the straightness of the path of growth of an axon, a variant of Mandelbrot's $(1977,1983)$ elegant fractal measures was used. (Fractals have been used to characterize the windiness of rivers and of coastlines.) The fractal dimension $D$ is the fractional dimension of a curve-here, it is the fractional dimension of the path of the axon in the plane. The formula used is:

$$
D=\log (L / a) / \log (d / a)
$$

where $D$ is the fractal dimension, $L$ is the total length of the path of the axon (the sum of the distances traveled in all of the 10-min intervals), $d$ is an estimate of the largest distance actually covered, and $a$ is the average length of a step or an observed interval of growth. ( $d$ should be a diameter of the potential area covered by a random path and is here estimated by taking the largest distance between any two points on the growth path.) In this way, a completely straight growth path gives a fractal dimension $D=1$, and a completely random walk gives a fractal dimension, $D=2$. Statistics must be determined for the logarithms of the fractal dimensions. (Further details, including a computer program for all calculations, can be found in Katz and George (1985).

Change in direction of growth cone movement. The overall direction of axon growth during a 10-min time interval was calculated by simple trigonometry from the $x, y$ coordinates at the beginning and at the end of the time period. The direction was expressed as an angle in relation to an arbitrary but fixed axis, and the change in direction of growth was then the change in this angle between successive 10-min intervals. To reveal any tendency for the axons to turn to one particular side, right-sided angle changes were arbitrarily called "positive" and left-sided angle changes were called "negative."

Change in axonal orientation. The orientation of the end of an axon was determined at the beginning and at the end of each 10-min time interval in relation to an arbitrary but fixed axis. The locations of the growth cone site and the neck site (or point $A$ when the growth cone neck had no bend) were taken as $x, y$ coordinates, and then the orientation of the end of the axon was computed as the arctangent of the difference in the $y$ values divided by the difference in the $x$ values. (A computer program containing this calculation, as standardized to an arbitrary axis, can be found in Katz et al., 1984.) 
The change in orientation was the change in this angle during a 10-min period. To reveal any tendency for the axons to turn to one particular side, right-sided deviations were arbitrarily called "positive" and left-sided deviations were called "negative."

Axonal bends. The overall bend in the terminal $40 \mu \mathrm{m}$ of an axon was computed for many axons at 10-min intervals. Three distances were used in this calculation: the distance $G A$ between the growth cone and point $A$, the distance $A C$ between point $A$ and point $C$, and the distance $G C$ between the growth cone and point $C$. The overall bend at point $A$ was taken to be the angle $A A$ (Fig. 2), where:

$$
A A=2 \arctan (R /(S-G C))
$$

and where:

$$
\begin{gathered}
S=(1 / 2)(G C+A C+G A) \\
R=((S-G C)(S-A C)(S-G A) / S)^{1 / 2}
\end{gathered}
$$

(This is the standard trigonometric formula for calculating an angle of a triangle when given the lengths of the three sides.) To reveal any tendency for the axons to bend to one particular side, right-sided bends were arbitrarily called "positive" and left-sided bends were called "negative."

Neck bends. The overall bend at the neck of the growth cone was computed in a similar manner for many axons at 10 -min intervals. Here, the three distances used in the calculation were: the distance between the center of the growth cone and the neck of the growth cone, the distance between the neck and point $A$, and the distance between the growth cone and point $A$. When there was no acute bend at the growth cone neck, it was not always possible to unequivocally distinguish the neck point. In these cases, the radius of curvature of the neck region was approximately the same as the radius of curvature of the terminal $40 \mu \mathrm{m}$ of axon, and the overall bend in the latter region was taken as an approximation of the bend at the neck region.
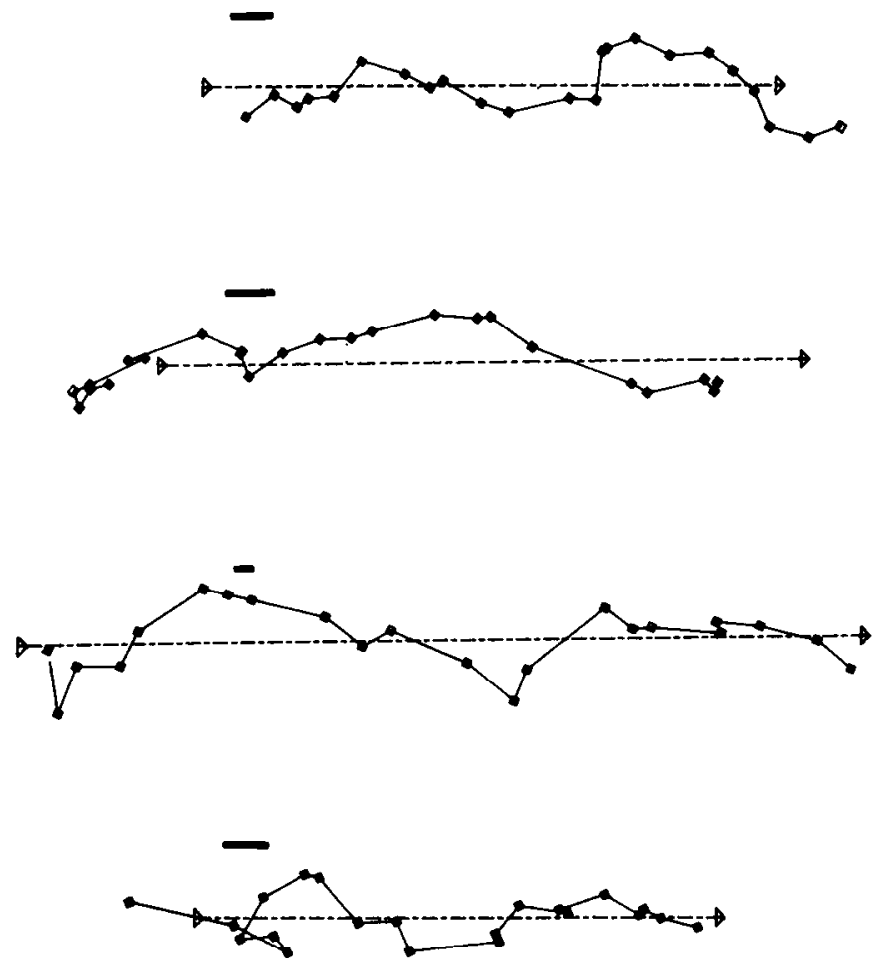

Figure 3. Computer reconstruction of growth paths of four typical axons in tissue culture, indicating their tendency to grow in fairly straight paths. Each square is the location of the growth cone at the end of a successive 10-min time interval. The dashed line with arrowheads indicates the principal component of growth. The top two axons are embryonic frog axons; the bottom two axons are embryonic chick dorsal root ganglion axons. Fractal dimensions for the axons are (from top to bottom): $D=1.06, D=1.05, D$ $=1.09$, and $D=1.13 . B a r=10 \mu \mathrm{m}$.

\section{Results}

\section{Description of axonal growth}

In real time, the elongation of an axon is difficult to perceive, although the growth cone movements are sufficiently fast to perceptibly change the shape of the axon tip. However, in time lapse, the axon can be seen to elongate in spurts and to undergo frequent short retractions. Although the growth cone sends off microprocesses in all directions, the major axonal elongation proceeds forward and the axon tends to maintain its same orientation (Fig. 3) (Katz et al., 1984).

The growth cone is continuously active. In our tissue culture system, the growth cones form long (6 to $10 \mu \mathrm{m})$, spike-shaped filopodia which can shoot out in any direction and which often form temporary attachments to the substrate. Frequently, these filopodia retract suddenly, and sometimes they "break" midway along their lengths at sharp angles, waving their tips in the media. Concurrently, the edges of the growth cone also give rise to short (2 to $3 \mu \mathrm{m}$ ) ruffle-shaped lamellopodia which form and disappear continually.

A 10 to $20-\mu \mathrm{m}$ length of the axon tip is quite active and continually varies in diameter. Usually the major growth cone is at the very distal end of the axon, but other regions of this active tip area occasionally give rise to the major growth cone. Growth cone activity can create temporary sharp bends in this 10 to $20 \mu \mathrm{m}$ length, but the sharp angles quickly (within minutes) dissipate and the tip usually has only a shallow radius of curvature.

\section{Quantitating the straightness of growth of an axon}

Axons do not grow in perfectly straight lines (Fig. 3), but do they grow randomly? To assess the relative randomness or straightness of growth of an axon objectively, axons were followed as they elongated, and a quantitative measure was used based on the work of Mandelbrot (1977, 1983) (Table I). One of Mandelbrot's (1977; 1983) measures, the fractal dimension, describes the complexity of a curve. For curves in a plane, such as axon growth paths in tissue culture, the fractal dimension $D$ can be made to vary between 1 (for a perfectly straight path) and approximately 2 (for a completely random path)

First, it was verified that the particular estimate of fractal dimensions that was used (see "Materials and Methods") did indeed exhibit these theoretical limits. Various axon paths were simulated on a computer, and it was found that straight line axon paths always had a fractal dimension $D=1$. For axon paths generated as limited random walks in a plane, the average fractal dimension was $D=$ $1.83(\mathrm{SD}=0.30$, range $=1.13$ to $3.20, N=501$ simulated axons, each followed for 19 ten-minute time intervals).

Next, the formula was applied to actual tissue culture data. (To produce statistically useful sample fractal dimensions, the number of time intervals for each axon must be 8 or more (Katz and George, 1985).) For frog axons, the average fractal dimension was $D=1.28$ $(S D=0.22$, range $=1.01$ to $1.84, N=23$ axons followed for an average of 14 ten-minute time intervals). For chick axons, the average fractal dimension was $D=1.31(S D=0.23$, range $=1.09$ to $2.00, N=17$ axons followed for an average of 21 ten-minute time

TABLE

Fractal dimensions, $D$

Differences between the mean $D$ are not significant $(p<0.65, t$ test on the logs of the data) for frogs and chicks. Differences between the mean $D$ are significant $(p<0.001, t$ test on the logs of the data) for frogs and random walks and for chicks and random walks.

\begin{tabular}{lcccc}
\hline & Mean $D$ & Mode & Range & $N$ \\
\hline Frog Axons & & & & \\
$\quad$ Glass & $1.28 \pm 0.22$ & 1.22 & $1.01-1.84$ & 23 \\
$\quad$ Polyornithine & $1.33 \pm 0.18$ & 1.31 & $1.14-1.72$ & 11 \\
Chick axons & $1.31 \pm 0.23$ & 1.25 & $1.09-2.00$ & 17 \\
Random walks & $1.83 \pm 0.30$ & 1.75 & $1.13-3.20$ & 501 \\
\hline
\end{tabular}


intervals). The fractal numbers show that real axon paths are quite straight and are not random walks in a plane. Sample fractal dimensions are lognormally distributed (not normally distributed); thus, standard statistics must be done on the logs of the values (Katz and George, 1985). The $t$ tests on the logs of the data demonstrated that the straightness of growth of the frog and the chick axons were statistically identical $(\rho<0.65)$ when grown on homogeneous glass surfaces and that neither type of axon grew randomly $(\rho<0.001)$.

As a test of the effect of substrate adhesivity on the straightness of growth, fractal dimensions were also calculated for frog axons grown on polyornithine-coated, acid-rinsed glass coverslips. Here, the average fractal dimension was $D=1.33$ (SD $=0.18$, range $=$ 1.14 to $1.72, N=11$ axons followed for an average of 18 ten-minute time intervals). The straightness of growth on polyornithine is statistically indistinguishable from the straightness of growth on uncoated acid-rinsed coverslips $(\rho<0.50, t$ test $)$.

\section{Changes in direction of growth cone movement}

For each 10-min interval of data collection, the change in the direction of growth cone movement was calculated. The mean and the standard deviations were similar for both types of axons studied. For frog axons, the mean change in growth direction was $72^{\circ}$ (SD $=56^{\circ}$, rangc $=180^{\circ}$ to $0^{\circ}, N=337$ ); for chicks, the mean was $77^{\circ}$ (SD $=59^{\circ}$, range $=180^{\circ}$ to $0^{\circ}, N=343$ ). The means did not differ significantly ( $\rho<0.2, t$ test), and histograms of the angle distributions for the two types of axons had similar shapes, but $\chi^{2}$ tests showed that the two distributions were significantly different at the $p<0.001$ level.

Figure 4 is a set of histograms of these angle distributions, when they have been assigned right and left signs (positive is right, negative is left). The values fall in symmetric curves centered at about $0^{\circ}$, indicating that the axons had an approximately equal probability of deviating to the right or to the left.

To assess the tendency of an axon tip to change its direction of growth in a consistent direction, the frequency of deviations to the same side in consecutive 10-min intervals was counted. Then, the actual numbers were compared with expected numbers that were calculated under the assumption of independence. This assumption presumes that the change of direction in one 10-min interval is not at all correlated with the change in direction in the previous $10-\mathrm{min}$ interval. The expected number is simply $N / 2-$ where $N$ is the total number of consecutive time intervals. For frogs, the axons changed direction to the same side in 107 of a possible 293 times. $\chi^{2}$ analyses show that this is statistically significant at the $p<0.001$

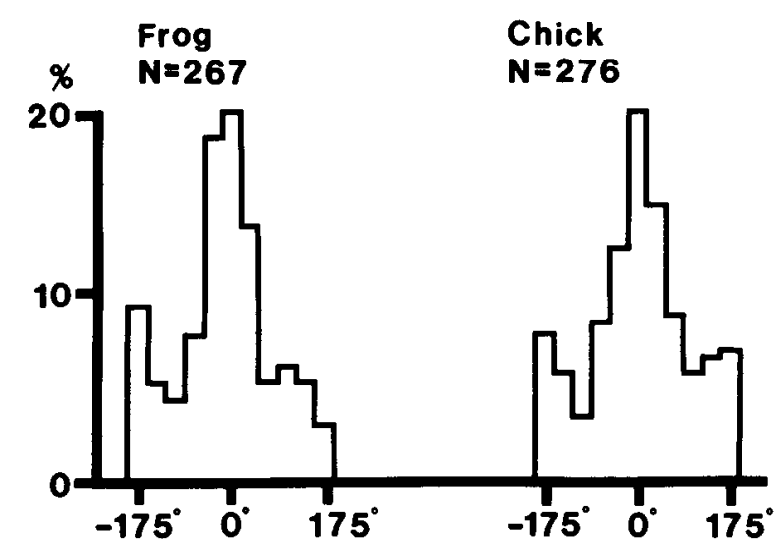

Figure 4. Histograms of changes in the direction of the growth cones of embryonic frog and chick axons grown on glass in dispersed tissue cultures. Growth cones can be found growing in any direction from the axon, but there is a tendency to grow at either moderately shallow angles or at very large angles (usually retractions; see Katz et al., 1984). level. Likewise, for chicks, 109 of a possible 253 consecutive changes were in the same direction, and this is statistically significant at the $p<0.025$ level. Thus, one must reject the hypothesis that the changes of growth direction are independent in each $10-\mathrm{min}$ interval. Instead, it appears that the growth cone actively alternates growth directions, because it changes its direction of growth more often than would be expected by pure chance.

\section{Changes in axon orientation}

For each 10-min interval of data collection, the change in the orientation of the end of an axon was calculated. The means and the standard deviations differed ( $p<0.001, t$ test) between the two types of axons: for frog axons, the mean change in orientation was $23^{\circ}\left(\mathrm{SD}=33.5^{\circ}\right.$, range $=132^{\circ}$ to $\left.0^{\circ}, N=337\right)$; for chicks, the mean was $15^{\circ}\left(\mathrm{SD}=16^{\circ}\right.$, range $=109^{\circ}$ to $\left.0^{\circ}, N=343\right)$. Histograms of the angle distributions for the two types of axons had similar shapes, but $\chi^{2}$ tests also showed that the two distributions were significantly different at the $p<0.025$ level. Another indication of this difference is that, in our culture systems, the range of angle deviations differed significantly between frogs and chicks. For frogs, the change in orientation of the tip of an axon was less than $75^{\circ}$ in $>95 \%$ of the 10 -min time periods. In contrast, for chicks the change in orientation of the tip of an axon was less than $50^{\circ}$ in $>95^{\circ}$ of the 10-min time periods.

Figure 5 is a set of histograms of these angle distributions, when they have been assigned right and left signs (positive is right, negative is left). The values fall in smooth bell-shaped curves centered at about $0^{\circ}$, indicating that the axons had an approximately equal probability of deviating to the right or to the left.

\section{Axonal bends}

The terminal $40 \mu \mathrm{m}$ of axon. The average degree of bend in the terminal $40 \mu \mathrm{m}$ of axon was computed for many individual observations separated by 10 -min time intervals. The results were identical for both types of axons: for frog axons, the mean bend was $17^{\circ}$ (SD $=20^{\circ}$, range $=111^{\circ}$ to $0^{\circ}, N=299$ ); for chick axons, the mean bend was $17^{\circ}\left(\mathrm{SD}=20^{\circ}\right.$, range $=116^{\circ}$ to $\left.0^{\circ}, N=296\right)$. In addition, histograms of the distributions of bends for the two types of axons had identical shapes, and $\chi^{2}$ tests showed that the two distributions were not significantly different $(p<0.10)$. Because the bend distributions for the frog axons could not be distinguished from the bend distributions for the chick axons, the data were pooled. For all axons, the mean bend in the terminal $40 \mu \mathrm{m}$ of axon was $17^{\circ}\left(\mathrm{SD}=20^{\circ}\right.$, range $=116^{\circ}$ to $0^{\circ}, N=595$ ). From the distribution histogram, one can calculate that, most of the time ( $>95 \%$ of cases), the bend in the terminal $40 \mu \mathrm{m}$ of an axon was less than $65^{\circ}$. (A qualitatively similar observation was reported by Gundersen and Park (1984).)

The distribution of bends is plotted as a single histogram in Figure 6 , where the bends have now been assigned right and left signs (positive is right, negative is left). The values fall in a smooth bellshaped curve centercd at about $0^{\circ}$, indicating that the axons were equally likely to bend to the right as to the left. (Gundersen and Barrett (1980) reported a similar result.)

To assess the tendency of an axon tip to continue bending in the same direction, the frequency of bends to the same side in consecutive 10-min intervals was counted. Then, the actual numbers were compared with expected numbers that were calculated under the assumption of independence. This assumption presumes that the direction of bend in one 10-min interval is not at all correlated with the direction of bend in the previous 10-min interval. (The expected number is simply $N / 2-$ where $N$ is the total number of consecutive time intervals.) For both frog axons and chick axons, it was found that axons continued bending to the same side in a disproportionately large number of cases, and $\chi^{2}$ analyses showed that this tendency was statistically significant $(p<0.01)$. Thus, one must reject the hypothesis that the direction of axonal bending is independent in each 10-min interval. Instead, it appears that, over 10 . 

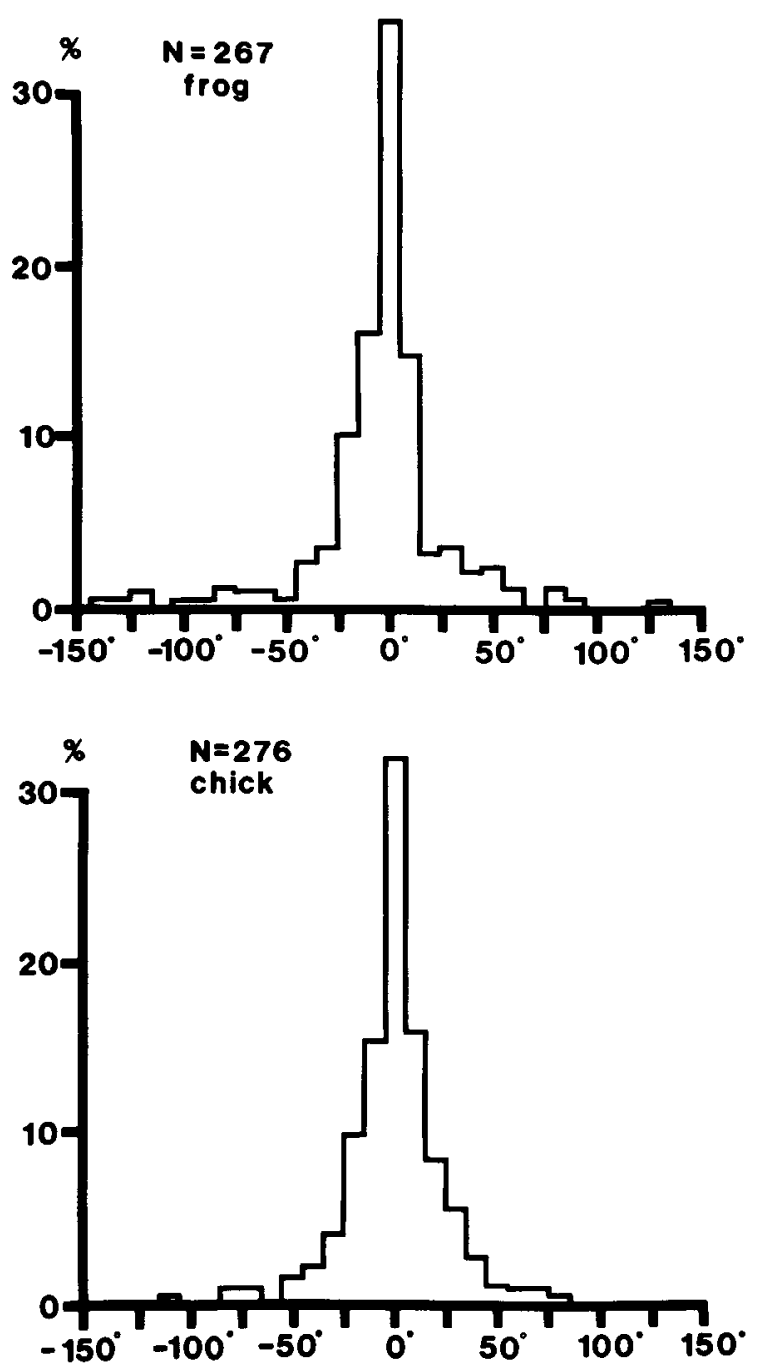

Figure 5. Histograms of changes in the orientation of embryonic frog and chick axons growing on glass in dispersed tissue cultures. Changes in axonal orientation were much more restricted than changes in the direction of growth cone movement (cf. Fig. 4). Most frog axons changed their orientation less than $75^{\circ}$ during a $10-\mathrm{min}$ time interval, and most chick axons changed their orientation less than $50^{\circ}$ during a 10 -min time interval.

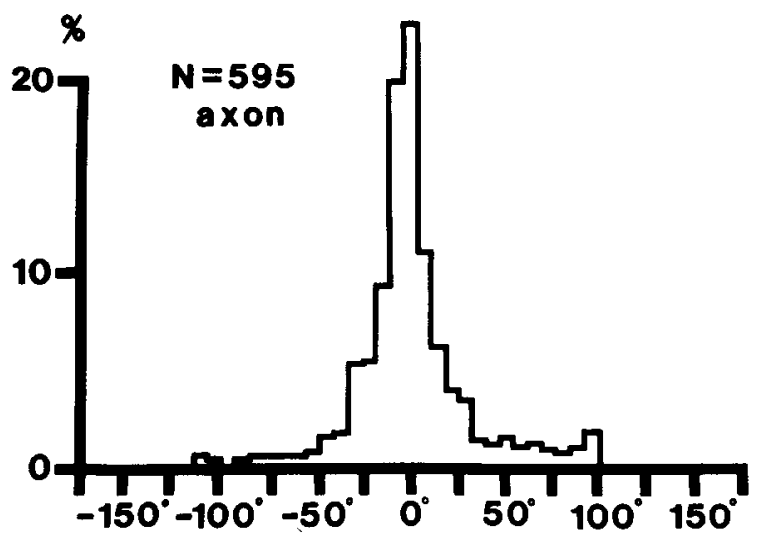

Figure 6. Histogram of the bends observed in the terminal $40 \mu \mathrm{m}$ of embryonic axons growing on glass in dispersed tissue cultures. The average bend was only $17^{\circ}$, and most of the time the bend was less than $65^{\circ}$. min intervals, the axon tends to bend or to remain bent to the same side more often than would be expected by pure chance. This result is consistent with the observation that the inherent straightening process in an axon can take as long as 10 or more minutes to complete (M. J. Katz, unpublished observations).

The growth cone neck region. The average degree of bend at the growth cone neck was computed for many individual observations separated by 10 -min time intervals. The results differed significantly ( $p<0.01, t$ test) between the two types of axons: for frog axons, the mean bend was $37^{\circ}\left(\mathrm{SD}=50^{\circ}\right.$, range $=179^{\circ}$ to $\left.0^{\circ}, N=299\right)$; for chick axons, the mean bend was $21^{\circ}\left(\mathrm{SD}=26^{\circ}\right.$, range $=176^{\circ}$ to $0^{\circ}, N=296$ ). Histograms of the distributions of bends for the two types of axons had similar shapes, but $\chi^{2}$ tests also showed that the two distributions were significantly different at the $p<0.001$ level.

For each type of axon, the distribution of neck bends is plotted as a histogram in Figure 7. For both types of axons, the values fall in smooth bell-shaped curves centered at approximately $0^{\circ}$, indicating that the axons were equally likely to bend to the right as to the left. From the distribution histograms, the bend at the frog growth cone neck was found to be less than $160^{\circ}>95 \%$ of the time, but for chick axons the neck bend was found to be less than $80^{\circ}>95 \%$ of the time. In other words, in these particular culture systems, the growth cones of frog axons tended to bend through twice the range of angles as did chick growth cones.

Another revealing measure is the correlation between the side to which the growlh cone itself is bent and the side to which the axon is bent. There was a clear tendency for the growth cone and the axon to be bent to the same side, but frequently the growth cone was bent in the opposite direction from the remainder of the axon. Specifically, the growth cone was bent in the opposite direction in $24 \%$ of the 308 separate observations. This demonstrates that bending at the growth cone neck can be independent from bending of the more proximal axon. The independence of bending in these two regions is also seen in the statistical analyses: by both the $t$ test and the $F$ test, the mean bend and the variance differed significantly between the two regions ( $p<0.001$ for frogs, $p<0.02$ for chicks).

\section{Discussion}

Detailed measurements confirm the impression that axons do not grow randomly, even in homogeneous environments, and the average fractal dirmension of $D=1.28$ to 1.33 shows that axons tend to grow straight (Fig. 3 and Table I). ${ }^{2}$ The determination of a specific objective number, the fractal dimension, that characterizes the relative straightness of growth of an axon now permits one to quantitatively assess the effect of various experimental perturbations (e.g., the use of cell motility-disrupting drugs such as taxol and cytochalasin) on this intrinsic tendency for straight growth (Katz and George, 1985).

One such critical variable is the substrate adhesivity. Substrate adhesivity has been clearly shown to affect the rate at which axons elongate (Luduena, 1973; Letourneau, 1975a; Collins, 1980), and regional differences in substrate adhesivity have been implicated as a determinant of the final patterns of axons (Weiss, 1945; Letourneau, 1975a; Collins and Garrett, 1980; Akers et al., 1981; Lander et al., 1982; Rogers et al., 1983; Katz and Lasek, 1985a, b). In contrast, comparisons of clongation on polyornithine-coated and uncoated acid-rinsed glass suggest that a homogeneously adhesive substrate may, in itself, have only a minor effect on the actual straightness of growth (Table I).

Interestingly, axons do not grow in convoluted, "wiggly" paths although their growth cones tend to actively alternate sides. (Recall that growth cones were found to change their directions of growth significantly more often than would be expected by pure chance.) Moreover, the angles through which growth cones changed their direction of growth spanned the entire range of a full $180^{\circ}$ (Fig. 4). This broad searching behavior of the growth cone appears to be strongly tempered by the axon proper, because it was found that 

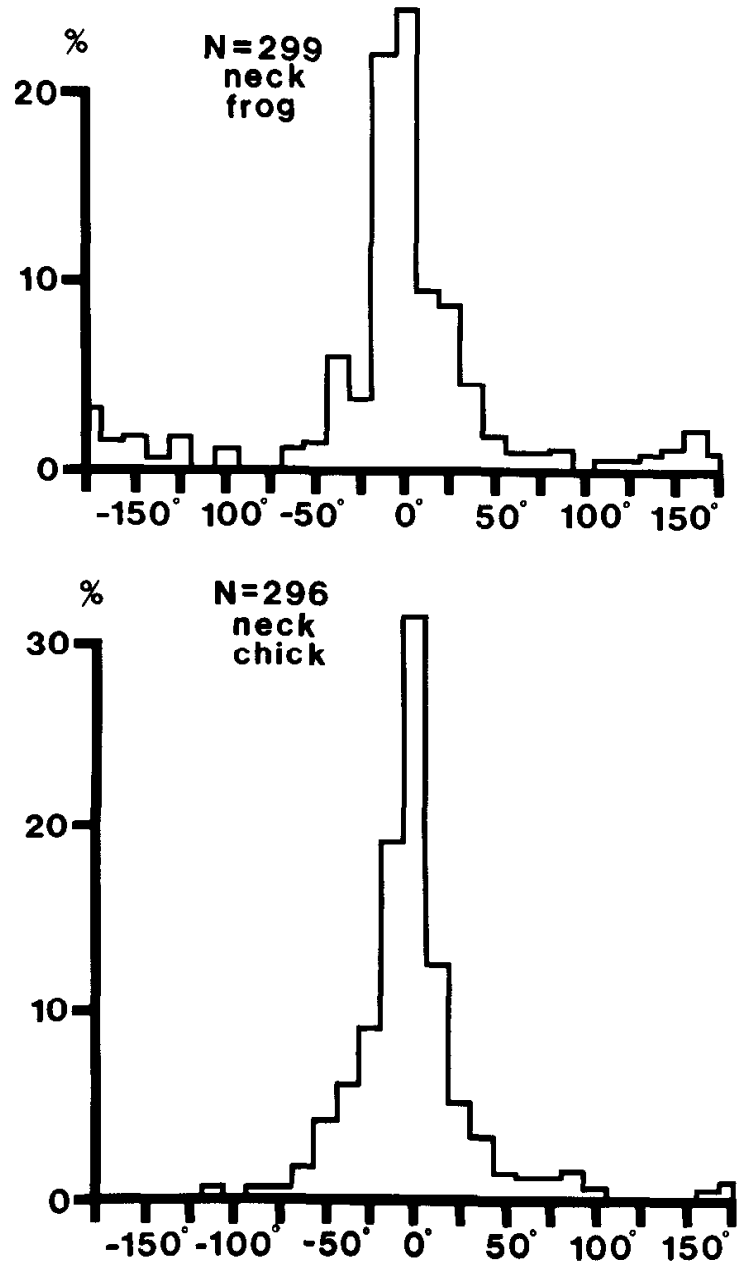

Figure 7. Histograms of the bends observed at the growth cone neck of embryonic frog and chick axons. The range of neck bends was significantly greater than the range of bends in the more proximal region of the axon (cf. Fig. 6).

the axon itself tends to maintain its same orientation (to continue pointing in the same direction) throughout its growth (Fig. 5).

One possiblc cause for the orienting and damping effects that the axon proper have on its route of growth is an intrinsic resistance to bending: the average axonal bend was only $17^{\circ}$, and $95 \%$ of the time, axons were found to be bent less than $65^{\circ}$ from straight lines. Axons appear to behave somewhat like elastic rods (M. J. Katz, unpublished observations), and the longitudinally oricnted axonal cytoskeleton may be the prime determinant of these rod-like properties of the axon (Gilbert, 1975; Morris and Lasek, 1982, 1984). In embryonic axons, the bulk of the core cytoskeleton appears to be formed of microtubules (Bray and Bunge, 1981; Letourneau, 1982), and microtubules have an inherent elastic stiffness (Kamimura and Takahashi, 1981). This longitudinal rigidity of the axonal cytoskeleton may be the major cause of the inherent tendency of axons to grow straight.

The intrinsic tendency for axons to grow straight again confirms the well documented notion that when axons grow in convoluted patterns extrinsic factors must be the major determinants of the final axonal configuration. As Harrison (1910, pp. 837/835) wrote:

\footnotetext{
${ }^{2}$ In contrast, in a mathematical analysis of fibroblasts in vitro, Gail and Boone (1970) reported that long-term ( $>5 \mathrm{hr}$ ) locomotion was random, with some tendency for the cells to persist in the same direction during the shortterm $(<2.5 \mathrm{hr})$. However, the authors pointed out that their test for randomness was not as sensitive as one might like.
}

The outgrowth of the nerve fibers. . .can be accounted for by power of growth in a straight line modified by deflection as a result of minor obstacles in the path. ... . . Given a form of protoplasma with power to extend itself in a definite direction so as to form a fiber, the next step is to determine the influences which may modify the direction of its growth and produce the specific arrangement of nerve tracts found in the mature organism.

At the other end of the spectrum, certain regions of the nervous system (such as the circumferential fibers of the spinal cord (Holley, 1983)) are characterized by sheets of parallel straight axons (Katz and Lasek, 1985a, b). In these regions, the intrinsic tendency of axons to grow straight may be one of the critical determinants of the final axon patterns.

\section{References}

Akers, R. M. D. F. Mosher, and J. E. Lilien (1981) Promotion of retinal neurite outgrowth by substratum-bound fibronectin. Dev. Biol. 86: 179-188.

Bray, D. (1973) Branching patterns of individual sympathetic neurons in culture. J. Cell Biol. 56: 702-712.

Bray, D. (1979) Mechanical tension produced by nerve cells in tissue culture. J. Cell Sci. 37: 391-410.

Bray, D., and M. B. Bunge (1981) Serial analysis of microtubules in cultured rat sensory axons. J. Neurocyto!. 10: 589-605.

Collins, F. (1980) Neurite outgrowth induced by the substrate associated material from nonneuronal cells. Dev. Biol. 79: 247-252.

Collins, F., and J. E. Garrett, Jr. (1980) Elongating nerve fibers are guided by a pathway of material released from embryonic nonneuronal cells. Proc. Natl. Acad. Sci. U. S. A. 77: 6226-6228.

Gail, M. H., and C. W. Boone (1970) The locomotion of mouse fibroblasts in tissue culture. Biophys. J. 10: 980-993.

Gilbert, D. S. (1975) Axoplasm architecture and physical properties as seen in the Myxicola giant axon. J. Physiol. (Lond.) 253: 257-301

Gundersen, R. W. and J. N. Barrett (1980) Characterization of the turning response of dorsal root neurites toward nerve growth factor. J. Cell Biol. 87: $546-554$.

Gundersen, R. W., and K. H. C. Park (1984) The effects of conditioned media on spinal neurites: Substrate-associated changes in neurite direction and adherence. Dev. Biol. 104: 18-27.

Hamburger, V. (1960) A Manual of Experimental Embryology (Revised Edition), University of Chicago Press, Chicago.

Harrison, R. G. (1910) The outgrowth of the nerve fiber as a mode of protoplasmic movement. J. Exp. Zool. 9: 787-848.

Heacock, A. M., and B. W. Agranoff (1977) Clockwise growth of neurites from retinal explants. Science 198: 64-66.

Holley, J. A. (1983) Development of the circumferential axonal pathway. PhD. thesis, Case Western Reserve University, Cleveland.

Kamimura, S., and K. Takahashi (1981) Direct measurement of the force of microtubule sliding in flagella. Nature 293: 566-568.

Katz, M. J. (1984) Stereotyped and variable growth of redirected Mauthner axons. Dev. Biol. 104: 199-209.

Katz, M. J., and E. B. George (1985) Fractals and the analysis of growth paths, Bull. Math. Biol., in press.

Katz, M. J., and R. J. Lasek (1979) Substrate pathways which guide growing axons in Xenopus embryos. J. Comp. Neurol. 183: 817-832.

Katz, M. J., and R. J. Lasek (1985a) How are the elemental axon patterns produced in the spinal cord? in Perspectives of Neuroscience. From Molecule To Mind, Y. Tsukada, ed., pp. 43-60, University of Tokyo Press, Tokyo.

Katz, M. J., and R. J. Lasek (1985b) Early axon patterns of the spinal cord: experiments with a computer. Dev. Biol., in press.

Katz, M. J., and L. F. Watson (1984) Intensifier for bodian stain of tissue sections and cell cultures. Stain Technol., in press.

Katz, M. J., E. B. George, and L. J. Gilbert (1984) Axonal elongation as a stochastic walk. Cell Motil. 4: 351-370.

Lander, A. D., D. K. Fujii, D. Gospodarowicz, and L. F. Reichardt (1982) Characterization of a factor that promotes neurite outgrowth: Evidence linking activity to a heparin sulfale proteoglycan. J. Cell Biol. 94: 574-585. Lasek, R. J., J. Metuzals, and I. R. Kaiserman-Abramof (1983) Cytoskeletons 
reconstituted in vitro indicate that neurofilaments contribute to the helical structure of axons. In Developing and Regenerating Vertebrate Nervous Systems, R. R. Markwald and A. D. Kenny, eds., pp. 1-18, Alan R. Liss, Inc., New York.

Letourneau, P. C. (1975a) Possible roles for cell-to-substratum adhesion in neuronal morphogenesis. Dev. Biol. 44: 77-91.

Letourneau, P. C. (1975b) Cell-to-substratum adhesion and guidance of axonal elongation. Dev. Biol. 44: 92-101.

Letourneau, P. C. (1982) Analysis of microtubule number and length in cytoskeletons of cultured chick sensory neurons. J. Neurosci. 2: 806814.

Luduena, M. A. (1973) Nerve cell differentiation in vitro. Dev. Biol. 33: 268284.

Mandelbrot, B. B. (1977) Fractals: Form, Chance, and Dimension, W. H Freeman \& Co., New York.

Mandelbrot, B. B. (1983) The Fractal Geometry Of Nature, W. H. Freeman \& Co., New York.

Morris, J. R., and R. J. Lasek (1982) Stable polymers of the axonal cytoskeleton: The axoplasmic ghost. J. Cell Biol. 92: 192-198.

Morris, J. R., and R. J. Lasek (1984) Monomer-polymer equilibria in the axon: Direct measurement of tubulin and actin as polymer and monomer in axoplasm. J. Cell Biol. 98: 2064-2076.

Nieuwkoop, P. D., and J. Faber (1967) Normal Table of Xenopus laevis (Daudin), Ed. 2, Elsevier-North Holland Publishing Co., Amsterdam.

Rakic, P. (1971) Neuron-glia relationship during granule cell migration in developing cerebellar cortex. A Golgi and electronmicroscopic study in
Macacus rhesus. J. Comp. Neurol. 141: 283-312.

Ramón y Cajal, S. (1911) Histologie du Système Nerveux de l'Homme des Vertébretes, Tomes I and II, A. Maloine, Paris.

Rogers, S. L., P. C. Letourneau, S. L. Palm, J. McCarthy, and L. T. Furcht (1983) Neurite extension by peripheral and central nervous system neurons in response to substratum-bound fibronectin and laminin. Dev. Biol. 98: 212-220.

Shaw, G., and D. Bray (1977) Movement and extension of isolated growth cones. Exp. Cell Res. 104: 55-62.

Silver, J., and R. L. Sidman (1980) A mechanism for the guidance and topographic patterning of retinal ganglion cell axons. J. Comp. Neurol. 189: 101-111.

Speidel, C. C. (1933) Studies of living nerves. II. Activities of ameboid growth cones, sheath cells, and myelin segments, as revealed by prolonged observation of individual nerve fibers in frog tadpoles. Am. J. Anat. 52: 179.

Spitzer, N. C., and J. E. Lamborghini (1976) The development of the action potential mechanism of amphibian neurons isolated in culture. Proc. Natl. Acad. Sci. U. S. A. 73: 1641-1645.

Strassman, R. J., P. C. Letourneau, and N. K. Wessells (1973) Elongation of axons in an agar matrix that does not support cell locomotion. Exp. Cell Res. 81: 482-487.

Trinkaus, J. P. (1984) Cells Into Organs, Ed. 2, Prentice-Hall, Englewood Cliffs, N.J.

Weiss, P. (1945) Experiments on cell and axon orientation in vitro: The role of colloidal exudates in tissue organization. J. Exp. Zool. 100: 353-386. 